\title{
The prognostic effect of DDX3 upregulation in distant breast cancer metastases
}

\author{
Marise R. Heerma van Voss ${ }^{1,2} \cdot$ Willemijne A. M. E. Schrijver ${ }^{1}$ Natalie D. ter Hoeve $^{1} \cdot$ Laurien D. Hoefnagel $^{1}$.

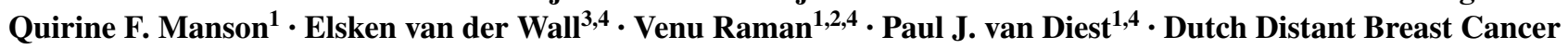 \\ Metastases Consortium
}

Received: 30 September 2016 / Accepted: 1 December 2016 / Published online: 20 December 2016

(C) The Author(s) 2016. This article is published with open access at Springerlink.com

\begin{abstract}
Metastatic breast cancer remains one of the leading causes of death in women and identification of novel treatment targets is therefore warranted. Functional studies showed that the RNA helicase DDX3 promotes metastasis, but DDX3 expression was never studied in patient samples of metastatic cancer. In order to validate previous functional studies and to evaluate DDX3 as a potential therapeutic target, we investigated DDX3 expression in paired samples of primary and metastatic breast cancer. Samples from 79 breast cancer patients with distant metastases at various anatomical sites were immunohistochemically stained for DDX3. Both cytoplasmic and nuclear DDX3 expression were compared between primary and metastatic tumors. In addition, the correlation between DDX3 expression and overall survival was assessed. Upregulation of cytoplasmic (28\%; OR $3.7 ; \mathrm{p}=0.002)$ was common in breast cancer metastases, especially in triple negative (TN) and high grade cases. High cytoplasmic DDX3 levels were most frequent in brain lesions (65\%)
\end{abstract}

Electronic supplementary material The online version of this article (doi:10.1007/s10585-016-9832-8) contains supplementary material, which is available to authorized users.

Paul J. van Diest

P.J.vanDiest@umcutrecht.nl

1 Department of Pathology, University Medical Center Utrecht, Utrecht, The Netherlands

2 Department of Radiology and Radiological Sciences, School of Medicine, Johns Hopkins University, Baltimore, MD, USA

3 Cancer Center, University Medical Center Utrecht, Utrecht, The Netherlands

4 Department of Oncology, School of Medicine, Johns Hopkins University, Baltimore, MD, USA and significantly correlated with high mitotic activity and triple negative subtype. In addition, worse overall survival was observed for patients with high DDX3 expression in the metastasis (HR 1.79, $\mathrm{p}=0.039$ ). Overall, we conclude that DDX3 expression is upregulated in distant breast cancer metastases, especially in the brain and in TN cases. In addition, high metastatic DDX3 expression correlates with worse survival, implying that DDX3 is a potential therapeutic target in metastatic breast cancer, in particular in the clinically important group of $\mathrm{TN}$ patients.

Keywords DEAD box RNA helicases - DDX3 .

DDX3X $\cdot$ Breast cancer $\cdot$ Metastasis $\cdot$ Brain metastasis

\author{
Abbreviations \\ DDX3 DEAD box RNA helicase 3 \\ MAI Mitotic activity index \\ ER Estrogen receptor \\ PR Progesterone receptor \\ HER2 Human epidermal growth factor receptor \\ OR Odds ratio \\ 95\% CI 95\% confidence interval \\ HR Hazard ratio \\ TN Triple negative \\ EMT Epithelial to mesenchymal \\ TMA Tissue microarray
}

\section{Background}

DDX3 (encoded by $D D X 3 X$ ) is a DEAD box RNA helicase with ATPase dependent helicase activity, which is involved in several steps of endogenous RNA metabolism and translation initiation [1-4]. DDX3 has been implicated in neoplastic transformation due to its role in cell cycle 
progression [5, 6] and its anti-apoptotic properties [7-9]. In addition, DDX3 has been shown to promote several steps of tumor metastasis. Overexpression of DDX3 resulted in increased motility and migration by induction of an epithelial-to-mesenchymal (EMT) phenotype with loss of E-cadherin $[10,11]$ and upregulation of Snail expression [12]. Furthermore, DDX3 was found to promote anchorage independent growth and invasive capacities of cancer cells through regulation of mRNA translation [13, 14]. DDX3 knockdown has also been shown to result in reduced breast cancer metastases in mice [15]. These findings have led to the development of DDX3 inhibitors for the treatment of breast cancer [15] among other malignancies [5, 6, 16, 17]. The tumor-enhancing role of DDX3 was corroborated by studies on DDX3 expression in patient samples of primary tumors [5, 6], but DDX3 expression was never studied in metastatic cancer samples.

Although therapeutic options for patients with metastatic breast cancer have increased, the vast majority of patients still develops resistance to treatment and eventually succumbs to the disease [18]. With 5-year survival rates of 25\% [19] and approximately 40,000 deaths on a yearly basis in the United States, metastatic breast cancer still ranks second on the list of causes of cancer deaths in women, accounting for $15 \%$ of all cancer deaths [20]. Therefore the identification of novel therapeutic targets that inhibit the development and outgrowth of breast cancer metastases remains urgently wanted.

Upregulation of DDX3 in metastases would confirm the role of DDX3 in metastatic tumor progression that has been suggested in functional studies. In addition, high DDX3 expression levels in metastatic lesions could indicate that breast cancer metastases are reliant on high DDX3 expression, and that patients with advanced disease could benefit from treatment with DDX3 inhibitors under development. Therefore, this study aimed to evaluate DDX3 expression in distant breast cancer metastases as compared to their primary tumor.

\section{Methods}

\section{Patient samples}

Tissue microarrays (TMAs) containing paired samples from 97 primary breast cancer and their distant metastases were previously assembled [21, 22]. All TMAs included multiple cores per patient. 18 pairs were incomplete due to damaged or detached cores during cutting or staining, or due to cores no longer containing invasive carcinoma. The TMA included metastases from various anatomical sites, including brain, skin, lung, liver, bone, ovaries, uterus and the gastro-intestinal tract. Clinicopathological data and follow up data were retrieved from the pathology reports and patient files. Overall survival was calculated from the time of diagnosis of the metastatic lesion. For this study only anonymous archival leftover pathology material was used. Therefore no informed consent is required according to Dutch legislation [23], as this use of redundant tissue for research purposes is part of the standard treatment agreement with patients in the UMC Utrecht [24].

\section{Immunohistochemistry}

Four $\mu \mathrm{m}$ thick sections were cut, mounted on Surgipathe $\mathrm{X}$-tra adhesive slides (Leica Biosystems, Milton Keynes, UK), deparaffinized in xylene and rehydrated in decreasing ethanol dilutions. Endogenous peroxidase activity was blocked with $1.5 \%$ hydrogen peroxide buffer for $15 \mathrm{~min}$ and was followed by antigen retrieval by boiling for $20 \mathrm{~min}$ in EDTA buffer (pH 9.0). Slides were blocked with protein block from Novolink Polymer Detection System (Leica Microsystems, Eindhoven, The Netherlands) and subsequently incubated in a humidified chamber for $1 \mathrm{~h}$ with anti-DDX3 (1:50, mAb AO196) [25]. Post primary block, secondary antibodies and diaminobenzidine treatment were performed with the same Novolink Polymer Detection System according to the manufacturer's instructions. The slides were lightly counterstained with hematoxylin and mounted. Appropriate positive and negative controls were used throughout.

Scoring was performed by consensus of two observers (PvD. and MHvV.). DDX3 shuttles between the nucleus and cytoplasm [26]. Since we previously observed distinct cytoplasmic and nuclear expression patterns, we allocated separate scores to cytoplasmic and nuclear DDX3 expression, as before [5]. Almost all cells expressed cytoplasmic DDX3, but the intensity varied and was therefore scored semi-quantitatively as absent (0), weak (1), moderate (2) or strong (3). The optimal cut-off point was selected using the online tool cut-off finder, which helps to select a cut-off that maximizes the difference in survival between groups [27]. Cases with score 0 to 2 were classified as having low DDX3 expression and evaluated against cases with high (score 3) expression, as before [6]. Cytoplasmic DDX3 was considered upregulated when DDX3 expression was low in the primary tumor (0-2) and high in the metastasis (3). Although the intensity of nuclear DDX3 in cells was similar, the fraction of positive cells varied. Therefore, the percentage of DDX3 positive nuclei was scored, regarding samples with $\geq 1 \%$ DDX3 staining as positive. When nuclear DDX3 was absent from the primary tumor and present in the metastasis, nuclear DDX3 was considered upregulated. 
Table 1 Changes in cytoplasmic DDX3 expression in breast cancers from primary to metastatic tumors at different sites

\begin{tabular}{lclclc}
\hline & $\mathrm{N}$ & \multicolumn{4}{l}{ Cytoplasmic DDX3 } \\
\cline { 3 - 6 } & & High to low & Low to high & OR $(95 \% \mathrm{CI})$ & p-value \\
\hline Total & 79 & $6(8 \%)$ & $22(28 \%)$ & $3.7(1.58-8.51)$ & 0.002 \\
Brain & 31 & $1(3 \%)$ & $15(48 \%)$ & $15.0(3.29-68.34)$ & $<0.001$ \\
Lung & 15 & $0(0 \%)$ & $3(20 \%)$ & - & 0.083 \\
Skin & 20 & $1(5 \%)$ & $4(20 \%)$ & $4.0(0.53-30.31)$ & 0.180 \\
Other & 13 & $4(31 \%)$ & $0(0 \%)$ & - & 0.046 \\
\hline
\end{tabular}

Paired odds ratio (OR) is calculated by taking the ratio of discrepant pairs. Paired p-values are calculated by McNemar's test

\section{Statistics}

Dichotomized cytoplasmic and nuclear DDX3 scores in primaries and metastases of the same patient were compared. Paired odds ratios were calculated by taking the ratio of discrepant pairs. p-values were calculated by McNemar's test. Correlations between high DDX3 in metastases and other clinicopathological variables were studied. Discrete variables were compared by $\chi^{2}$ or Fisher's exact test. The distribution of continuous variables was assessed graphically and Student's $t$ tests or Mann Whitney U-tests were used for normally and non-normally distributed variables, respectively. Overall survival data from the time of biopsy of metastatic lesions onward was available for 58 patients and compared between patients with low verses high metastatic DDX3 expression by plotting Kaplan-Meier curves and performing modified Wilcoxon tests. Potential confounders were analyzed by including variables associated with both DDX3 expression and survival in a multivariate cox-proportional hazards model. Effect measure modification was explored by including multiplicative interaction terms in a Cox proportional-hazards model. If sample size allowed stratified analysis was performed in the case of significant interaction. P-values smaller than 0.05 were considered statistically significant. All statistical analyses were performed with $\mathrm{R}$ version 3.2.0.

\section{Results}

\section{DDX3 is overexpressed in breast cancer metastases}

DDX 3 could be assessed in 79 paired primary breast cancers and corresponding metastases. High cytoplasmic DDX3 expression was observed in 19\% of primary breast cancers and 39\% of metastases. Pairwise analysis of primary tumors and metastasis in the same patient showed that $28 \%$ of metastases had upregulated DDX3 expression, whereas DDX3 was downregulated in only $8 \%$ of patients (Table 1). This difference was highly statistically significant with an OR of 3.7 (95\% CI 1.58-8.51; $\mathrm{p}=0.002$ ). Figure 1 shows examples of increased cytoplasmic DDX3 expression at different metastatic sites. DDX3 expression was especially prominent in breast cancer brain metastases, with $65 \%$ of metastases having high DDX3 expression and $48 \%$ of patients having an increase as compared to their primary tumor (OR 15.0, 95\% CI 3.29-68.34, $\mathrm{p}<0.001$, Table 1). Upregulation of cytoplasmic DDX3 expression was also common in lung $(20 \%)$ and skin $(20 \%)$ metastases. The low number of available liver $(n=3)$ and bone $(n=3)$ metastases did not allow subgroup analysis for these specific anatomical sites.

Nuclear DDX3 expression was observed in $22 \%$ of primary breast cancers and $13 \%$ of metastases. As shown in Table 2, conversion from nuclear DDX3 from absent in the primary tumor to present in the metastasis occurred in $9 \%$ of pairs, whereas the opposite occurred in $18 \%$ of patients (OR 0.5 ; 95\% CI $0.21-1.22$; $\mathrm{p}=0.127$ ).
Primary Breast Cancer
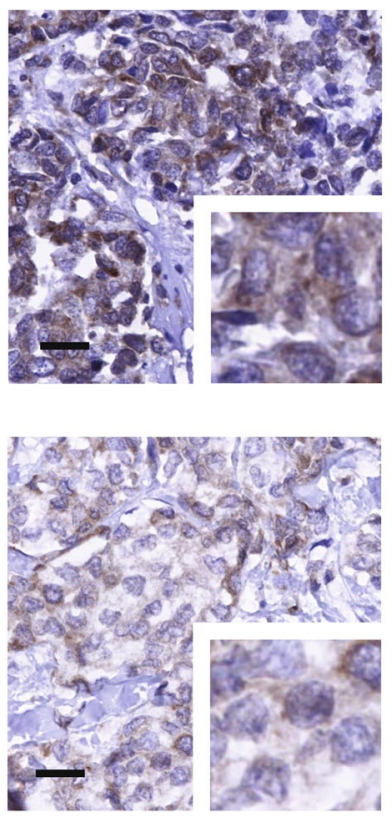

Corresponding Metastasis

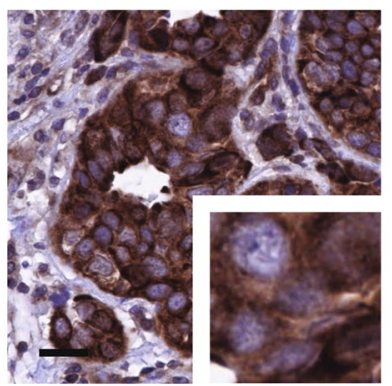

Brain

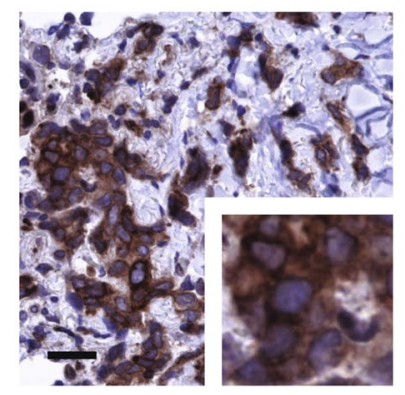

Lung
Fig. 1 Cytoplasmic DDX3 expression is upregulated in breast cancer metastases. Examples of upregulation of cytoplasmic DDX3 expression in breast cancer metastases at different anatomical locations as compared to the originating primary breast cancer in the same patient. Analysis was performed in 79 pairs, $\times 40$ magnification, scale bar indicates $25 \mu \mathrm{m}$ 
Table 2 Changes in nuclear DDX3 expression in breast cancers from primary to metastatic tumors at different sites

\begin{tabular}{llllll}
\hline & $\mathrm{N}$ & \multicolumn{2}{l}{ Nuclear DDX3 } & & \\
\cline { 3 - 6 } & & $\begin{array}{l}\text { Present to } \\
\text { absent }\end{array}$ & $\begin{array}{l}\text { Absent to } \\
\text { present }\end{array}$ & OR (95\% CI) & p-value \\
\hline Total & 79 & $14(18 \%)$ & $7(9 \%)$ & $\begin{array}{c}0.5(0.21- \\
1.22)\end{array}$ & 0.127 \\
Brain & 31 & $5(16 \%)$ & $3(10 \%)$ & $\begin{array}{c}0.6(0.15- \\
2.47)\end{array}$ & 0.480 \\
Lung & 15 & $3(20 \%)$ & $0(0 \%)$ & - & 0.083 \\
Skin & 20 & $5(25 \%)$ & $2(10 \%)$ & $\begin{array}{l}0.4(0.08- \\
1.95)\end{array}$ & 0.257 \\
Other & 13 & $1(8 \%)$ & $2(15 \%)$ & $2.0(0.19-$ & 0.564 \\
& & & & $21.04)$ & \\
\hline
\end{tabular}

Paired odds ratio (OR) is calculated by taking the ratio of discrepant pairs. Paired p-values are calculated by McNemar's test

\section{Metastatic cytoplasmic DDX3 overexpression correlates with triple-negative receptor status and high mitotic activity}

In order to catalogue what characterized patients with high metastatic DDX3, we explored correlations with other clinicopathological characteristics (Table 3). High cytoplasmic DDX3 in metastasis was associated with a higher mitotic activity index (MAI) (30.3 vs. $21.3 ; \mathrm{p}=0.033)$ and triplenegative molecular subtype ( $43 \%$ vs. $24 \% ; \mathrm{p}=0.019$ ) in the primary tumor and negative estrogen receptor (ER) status in the metastasis ( $72 \%$ vs. $45 \%$; $=0.043$ ). Since DDX3 in metastases was associated with ER-negativity and possible negative selection pressure occurred on ER expression when patients were treated with hormonal treatment, we assessed whether adjuvant treatment of the primary tumor correlated with metastatic DDX3 expression. No correlation was found between high DDX3 in metastases and chemotherapy ( $47 \%$ vs. $54 \% ; \mathrm{p}=0.795$ ), hormonal therapy $(21 \%$ vs $27 \% ; p=0.790)$ or treatment with trastuzamab (3\% vs. $0 \% ; \mathrm{p}=1)$. Nuclear DDX3 in metastases was associated with negative HER2 receptor status in the metastasis, but did not correlate with other clinicopathological variables (supplementary table 1).

\section{Metastatic DDX3 expression correlates with worse survival}

We performed survival analysis to see whether DDX3 expression correlated with clinical outcome in metastatic breast cancer patients (Fig. 2). Median overall survival after the metastatic lesion was biopsied was shorter in patients with high cytoplasmic DDX3 (11.18 months) when compared to patients with low cytoplasmic DDX3 (20.14 months; HR 1.79; 95\% CI 0.97-3.33; $\mathrm{p}=0.039$ ). Because the molecular subtype of the primary tumor and the location and ER-status of the metastasis were associated with both high cytoplasmic DDX3 and survival, potential confounding by these factors was explored in a multivariate model as much as sample size permitted. The association between cytoplasmic DDX3 and survival weakened after adjustment for individual covariates by Cox-regression analysis. This indicates that molecular subtype $\left(\mathrm{HR}_{\text {adjusted }}\right.$ 1.38; 95\% CI 0.73-2.63; $\mathrm{p}=0.324)$, ER status $\left(\mathrm{HR}_{\text {adjusted }}\right.$ $1.51 ; 95 \%$ CI $0.81-2.82 ; \mathrm{p}=0.200)$ and location of the metastasis $\left(\mathrm{HR}_{\text {adjusted }} 1.52 ; 95 \% \mathrm{CI} 0.9-2.94 ; \mathrm{p}=0.210\right)$ are confounding the relation between cytoplasmic DDX3 and survival.

In addition, a significant correlation between the presence of nuclear DDX3 in metastases and overall survival was observed. Patients with nuclear DDX3 had a shorter median survival of 8.8 vs. 19.4 months (HR 3.28; 95\% CI $1.23-8.75 ; \mathrm{p}=0.034)$. Unfortunately multivariate analysis was not possible due to the low number of patients with nuclear DDX3 in the metastasis. Overall we conclude that there is a relation between metastatic DDX3 expression and survival, which for cytoplasmic DDX3 can in part be attributed to the molecular subtype and location of these tumors.

\section{Discussion}

DDX3 is an RNA helicase with oncogenic properties, which has been found to promote metastasis in functional studies. However, DDX3 expression had never been specifically evaluated in metastatic cancer patient samples. In this study, we therefore compared DDX3 expression in primary breast cancers to that in corresponding distant metastatic lesions. Cytoplasmic DDX3 expression was significantly higher in metastatic cancer samples, especially in brain metastases and triple negative cases. In addition, there is a correlation between DDX3 expression in the metastasis and worse overall survival in patients with metastatic breast cancer.

Previous studies have indicated that DDX3 overexpression facilitates dissemination of cancer cells through induction of an EMT phenotype [10-12]. Increased motility and anchorage independent growth have also been linked to the role DDX3 has in mRNA translation. Chen, et al. found DDX3 to increase invasive properties through a direct role in Rac1 translation, which in its turn stabilizes $\beta$-catenin expression resulting in activated Wnt-signaling [14]. Furthermore, Hagerstrand, et al. found that DDX3 mediates IRES-dependent translation, resulting in increased anchorage independent growth in cancers with 3q26 amplification. In addition to promoting the dissemination process, our finding that among patients with established metastases, those with DDX3 expression have worse overall survival 
Table 3 Correlation between cytoplasmic DDX3 expression and clinicopathological variables in breast cancer metastases

\begin{tabular}{|c|c|c|c|c|}
\hline & \multicolumn{4}{|c|}{ Cytoplasmic DDX3 } \\
\hline & Total & Low & High & p-value \\
\hline \multicolumn{5}{|c|}{ Characteristics primary tumor } \\
\hline $\begin{array}{l}\text { Tumor size in } \\
\mathrm{cm}, \text { median } \\
\text { (IQR) }\end{array}$ & $7(2)$ & $7(2.75)$ & $7(2)$ & $0.657^{\#}$ \\
\hline \multicolumn{5}{|l|}{ Histology, n (\%) } \\
\hline Ductal & $67(86)$ & $39(81)$ & $28(90)$ & $0.857^{* *}$ \\
\hline Lobular & $8(10)$ & $6(13)$ & $2(6)$ & \\
\hline Metaplastic & $3(4)$ & $2(4)$ & $1(3)$ & \\
\hline Apocrine & $1(1)$ & $1(2)$ & 0 & \\
\hline \multicolumn{5}{|l|}{ Grade, n (\%) } \\
\hline I & $1(1)$ & $1(2)$ & 0 & $0.670^{* *}$ \\
\hline II & $21(27)$ & $14(30)$ & $7(23)$ & \\
\hline III & $55(71)$ & $31(67)$ & $24(77)$ & \\
\hline Missing & 2 & 2 & 0 & \\
\hline MAI, mean (SD) & $24.8(19.7)$ & $21.3(18.6)$ & $30.3(20.5)$ & $0.033^{\$}$ \\
\hline \multicolumn{5}{|l|}{ Lympnodes, n (\%) } \\
\hline Negative & $39(49)$ & $28(58)$ & $11(35)$ & $0.080^{*}$ \\
\hline Positive & $40(51)$ & $20(42)$ & $20(65)$ & \\
\hline Age, mean (SD) & $52.2(11.0)$ & $54.0(11.0)$ & $49.5(10.4)$ & $0.074^{\$}$ \\
\hline Missing & 1 & 1 & 0 & \\
\hline \multicolumn{5}{|c|}{ Molecular subtype, n (\%) } \\
\hline HER2-enriched & $11(15)$ & $5(12)$ & $6(20)$ & $0.019 * *$ \\
\hline Luminal A & $29(41)$ & $23(56)$ & $6(20)$ & \\
\hline Luminal B & $8(11)$ & $3(7)$ & $5(17)$ & \\
\hline Triple negative & $23(32)$ & $10(24)$ & $13(43)$ & \\
\hline Missing & 8 & 7 & 1 & \\
\hline \multicolumn{5}{|c|}{ Characteristics metastasis } \\
\hline \multicolumn{5}{|l|}{ Location, $\mathrm{n}(\%)$} \\
\hline Brain & $31(39)$ & $11(23)$ & $20(65)$ & $0.001 *$ \\
\hline Skin & $20(25)$ & $15(31)$ & $5(16)$ & \\
\hline Lung & $15(19)$ & $10(20)$ & $5(16)$ & \\
\hline Other & $13(16)$ & $12(25)$ & $1(3)$ & \\
\hline \multicolumn{5}{|c|}{ Estrogen receptor, n (\%) } \\
\hline Negative & $39(57)$ & $18(45)$ & $21(72)$ & $0.043^{*}$ \\
\hline Positive & $30(43)$ & $22(55)$ & $8(28)$ & \\
\hline Missing & 10 & 8 & 2 & \\
\hline \multicolumn{5}{|c|}{ Progesterone receptor, n (\%) } \\
\hline Negative & $39(57)$ & $26(67)$ & $22(81)$ & $0.295^{*}$ \\
\hline Positive & $30(43)$ & $13(33)$ & $5(19)$ & \\
\hline Missing & 13 & 9 & 4 & \\
\hline \multicolumn{5}{|c|}{ HER2 receptor, n (\%) } \\
\hline Negative & $48(73)$ & $31(82)$ & $20(69)$ & $0.363^{*}$ \\
\hline Positive & $18(27)$ & $7(18)$ & $9(31)$ & \\
\hline Missing & 12 & 10 & 2 & \\
\hline \multicolumn{5}{|l|}{ Nuclear DDX3, n (\%) } \\
\hline Absent & $69(87)$ & $42(79)$ & $27(26)$ & $1 * *$ \\
\hline Present & $10(13)$ & $6(21)$ & $4(74)$ & \\
\hline
\end{tabular}

P-value calculated by *Chi square test, **Fisher exact test, "MannWhitney U test, ${ }^{\$}$ student's $t$ test is suggestive of DDX3 also providing a survival benefit to cancer cells after colonization of the metastatic niche. However, this difference can also be partly attributed to the frequent triple negative phenotype and brain localization of metastases with high cytoplasmic DDX3 expression. Notably, there are some contradictory reports in literature pointing towards DDX3 functioning as a tumor suppressor $[28,29]$. It is possible that the role of DDX3 in oncogenesis differs between genetic backgrounds and cancer types [30].

The mechanisms behind cytoplasmic overexpression and nuclear retention of DDX3 remain largely to be elucidated. Mutations in DDX3 have been detected in medulloblastomas [31], head and neck cancers [32] and hematological malignancies [33, 34], but were not identified in breast cancers [35]. In addition, there is no amplification of the DDX3 locus in DDX3 overexpressing breast cancer cell lines [10]. With regard to nuclear retention of DDX3, we know that DDX3 is exported out of the nucleus as part of messenger ribonucleoprotein complexes [2, 26, 36]. In the nucleus, DDX3 was previously found to localize to the nucleolus [37] where ribosomal assembly takes place, suggesting that nuclear DDX3 retention in metastases possibly reflects increased demand in protein synthesis. More research to further clarify the mechanisms of DDX3 overexpression and nuclear retention is needed.

We found a particularly large increase in cytoplasmic DDX 3 expression rates in brain metastases. Brain metastases are more common in patients with triple negative or HER2 amplified primary breast cancers [38], which have relatively high DDX3 expression. However, discordance rates for DDX3 were much higher (48\% upregulation) than for HER2 (2\%) and estrogen receptor (13\%) [21]. It is therefore hard to explain the DDX3 upregulation in brain metastases rates solely by an association with these molecular subtypes. Several other biological signatures have been found to characterize brain metastases. Wnt signaling mediates metastasis to the brain in both lung [39] and breast cancer [40]. DDX3 is a multilevel activator of the Wnt-signaling pathway $[5,6,14,41]$ and therefore potentially facilitates brain colonization in a Wnt-mediated fashion. Another feature of brain metastases is the expression of DNA repair genes [42, 43]. Inhibition of DDX3 reduced non-homologous end joining, a double strand break repair mechanism [5], implying that the high DDX3 levels in brain metastases could reflect a DNA damage response. Furthermore, overexpression of hypoxia-inducible factor $1 \alpha$ is common in brain metastases [44] and also associated with DDX3 expression in breast cancer [45]. However, metastatic DDX3 expression did not correlate with expression of the HIF- $1 \alpha$ target genes carbonic anhydrase IX (CAIX) and Glucose transporter 1 (GLUT-1; data not shown), making it unlikely that high DDX3 expression in brain metastases 


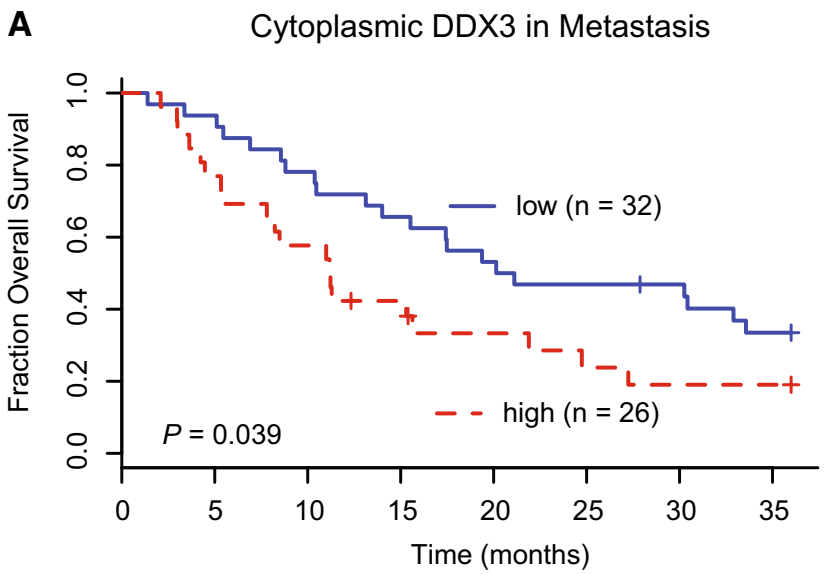

Fig. 2 Metastatic DDX3 is associated with shorter survival in distant breast cancer metastases. a Kaplan-Meier curves showing overall survival after biopsy of the metastasis in breast cancer patients with high cytoplasmic $(n=26)$ as compared to those with low cytoplasmic $(n=32)$ DDX3 expression in the metastatic lesion. b Kaplan-

is hypoxia-mediated. Last, metastatic niches differ also by the bioenergetic profile they impose on cells [46]. Brain metastases were demonstrated to upregulate glycolysis and oxidative phosphorylation capacity [47] and to have increased hexokinase 2 expression [48]. An additional reason for brain metastases to elevate DDX3 expression could be that DDX3 supports metabolic adaptation of cancer cells to the microenvironment of the brain. Although liver and bone metastases are also common in breast cancer patients, limited availability of tissue from these sites did not allow for subgroup analysis.

Besides biological relevance, high DDX3 expression in breast cancer metastases has potential clinical applications. Metastatic breast cancer, especially localized in the brain, is associated with short patient survival and severely impaired quality of life. Cerebral metastases occur early in triple negative cases [49], where the systemic therapeutic arsenal is particularly lacking. High DDX3 expression could serve as a therapeutic target in these patients. There are several small molecule inhibitors of DDX3 currently under development [50]. Although diffusion of these compounds over an intact blood brain barrier is limited [5], the small size of the inhibitors and the compromised blood brain barrier in brain metastases [51] potentially do allow for therapeutic levels to be reached. The DDX3 inhibitor RK-33 has potent radiosensitizing abilities [10], which could enhance the effect of whole brain radiation to treat brain metastases. Furthermore, given the role of DDX3 early in the metastatic process, DDX3 inhibitors could potentially also be used to prevent the emergence of metastases. At last, evaluation of DDX3 expression in patient samples could serve both as a prognostic biomarker and facilitate selection of those patients benefiting most from DDX3 inhibitors.
B

Nuclear DDX3 in Metastasis

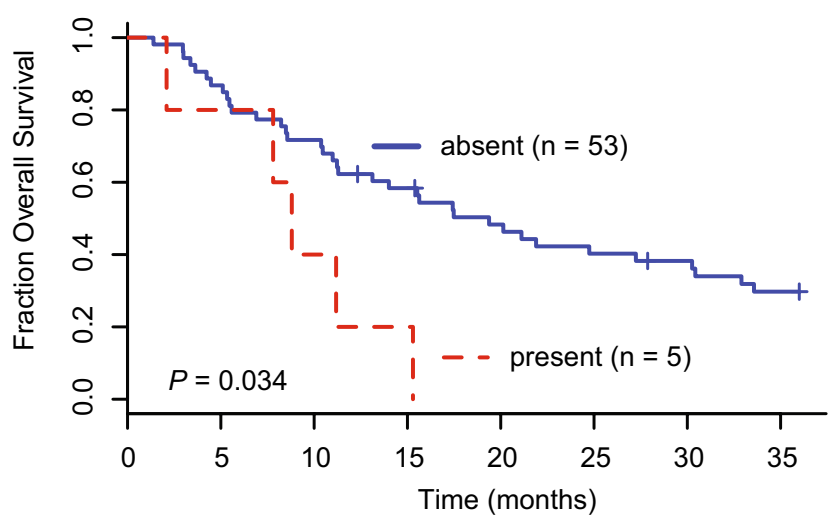

Meier curves showing overall survival after biopsy of the metastasis in breast cancer patients with nuclear DDX3 $(n=53)$ as compared to those without nuclear DDX3 $(n=5)$ in the metastatic lesion. P-values calculated by a modified Wilcoxon test

\section{Conclusions}

Cytoplasmic DDX3 expression is increased in breast cancer metastases, especially those located in the brain and occurring in triple negative cases. In addition, patients with high DDX3 levels in the metastatic lesion have shorter overall survival, implying that DDX3 is a potential therapeutic target in metastatic breast cancer.

Acknowledgements The Dutch Breast Cancer Consortium included Department of Pathology, University Medical Center Utrecht, Department of Pathology, Academic Medical Center, Amsterdam; Department of Pathology, Medical Center Alkmaar; Department of Pathology, Medical Center Zaandam; Department of Pathology, Radboud University Nijmegen Medical Center, Nijmegen; Department of Pathology, Canisius Wilhelmina Hospital, Nijmegen; Department of Pathology, VU University Medical Center, Amsterdam; Department of Pathology, The Netherlands Cancer Institute, Amsterdam; Laboratory for Pathology, Dordrecht; Department of Pathology, University Medical Center Groningen; Department of Pathology, St Antonius Hospital, Nieuwegein; Department of Pathology, Diakonessenhuis, Utrecht, Isala klinieken, Zwolle; Erasmus Medical Center Rotterdam; Gelre Hospital Apeldoorn, Laboratory Sazinon, Hoogeveen, and the Laboratory for Pathology Oost Nederland, Enschede, all in The Netherlands.

Funding This work was financially supported by Utrecht University Alexandre Suerman Stipend (MHVV), the Dutch Cancer Foundation (UU2013-5851; MHVV), Flight Attendant Medical Research Institute (VR) and Safeway (VR).

\section{Compliance with ethical standards}

Conflict interests Paul van Diest and Venu Raman have applied for a patent for the use of DDX3 as a cancer biomarker and are on the advisory board of Natsar Pharmaceuticals. 
Ethics approval and consent to participate For this study only anonymous archival leftover pathology material was used. Therefore no formal consent is required according to Dutch legislation [23], as this use of redundant tissue for research purposes is part of the standard treatment agreement with patients in our hospitals [24]. The medical research ethics committee of the UMC Utrecht confirmed that official approval of this study is not required (reference number WAG/ $\mathrm{mb} / 16 / 029330)$

Open Access This article is distributed under the terms of the Creative Commons Attribution 4.0 International License (http:// creativecommons.org/licenses/by/4.0/), which permits unrestricted use, distribution, and reproduction in any medium, provided you give appropriate credit to the original author(s) and the source, provide a link to the Creative Commons license, and indicate if changes were made.

\section{References}

1. Deckert $\mathbf{J}$ et al (2006) Protein composition and electron microscopy structure of affinity-purified human spliceosomal B complexes isolated under physiological conditions. Mol Cell Biol 26:5528-5543

2. Lai MC, Lee YH, Tarn WY (2008) The DEAD-box RNA helicase DDX3 associates with export messenger ribonucleoproteins as well as tip-associated protein and participates in translational control. Mol Biol Cell 19:3847-3858

3. Kasim V et al (2013) Determination of the role of DDX3 a factor involved in mammalian RNAi pathway using an shRNA-expression library. PloS One 8:e59445

4. Lee CS et al (2008) Human DDX3 functions in translation and interacts with the translation initiation factor eIF3. Nucleic Acids Res 36:4708-4718

5. Bol GM et al (2015) Targeting DDX3 with a small molecule inhibitor for lung cancer therapy. EMBO Mol Med 7:648-669

6. Heerma van Voss MR et al (2015) Identification of the DEAD box RNA helicase DDX3 as a therapeutic target in colorectal cancer. Oncotarget 6:28312-28326

7. Li Y et al (2006) Inducible resistance of tumor cells to tumor necrosis factor-related apoptosis-inducing ligand receptor 2-mediated apoptosis by generation of a blockade at the death domain function. Cancer Res 66:8520-8528

8. Sun M et al (2008) Identification of an antiapoptotic protein complex at death receptors. Cell Death Differ 15:1887-1900

9. Shih JW et al (2012) Critical roles of RNA helicase DDX3 and its interactions with eIF4E/PABP1 in stress granule assembly and stress response. Biochem J 441:119-129

10. Botlagunta $\mathrm{M}$ et al (2008) Oncogenic role of DDX3 in breast cancer biogenesis. Oncogene 27:3912-3922

11. Nozaki K et al (2014) DDX3X induces primary EGFR-TKI resistance based on intratumor heterogeneity in lung cancer cells harboring EGFR-activating mutations. PLoS One 9:e111019

12. Sun M et al (2011) The role of DDX3 in regulating Snail. Biochim Biophys Acta 1813:438-47

13. Hagerstrand D et al (2013) Systematic interrogation of $3 q 26$ identifies TLOC1 and SKIL as cancer drivers. Cancer Discov 3:1044-1057

14. Chen HH et al (2015) DDX3 modulates cell adhesion and motility and cancer cell metastasis via Rac1-mediated signaling pathway. Oncogene 34:2790-2800

15. Xie $\mathrm{M}$ et al (2015) NZ51, a ring-expanded nucleoside analog, inhibits motility and viability of breast cancer cells by targeting the RNA helicase DDX3. Oncotarget 6:29901-29913
16. Xie $M$ et al (2016) RK-33 radiosensitizes prostate cancer cells by blocking the RNA helicase DDX3. Cancer Res. doi:10.1158/0008-5472.CAN-16-0440

17. Wilky BA et al (2016) RNA helicase DDX3: a novel therapeutic target in Ewing sarcoma. Oncogene 35:2574-2583

18. Zeichner SB et al (2015) Survival of patients with de-novo metastatic breast cancer: analysis of data from a large breast cancerspecific private practice, a university-based cancer center and review of the literature. Breast Cancer Res Treat 153:617-624

19. Steeg PS (2016) Targeting metastasis. Nat Rev Cancer $16: 201-218$

20. Society AC (2015) Cancer facts \& figures. American Cancer Society, Atlanta

21. Hoefnagel LD et al (2010) Receptor conversion in distant breast cancer metastases. Breast cancer res 12: R75

22. Jiwa LS et al (2014) Upregulation of Claudin-4, CAIX and GLUT-1 in distant breast cancer metastases. BMC Cancer 14:864

23. The Medical Research Involving Human Subjects Act, 1998 [In Dutch: Wet medisch-wetenschappelijk onderzoek met mensen, WMO]. Burgerlijk Wetboek.

24. van Diest PJ (2002) No consent should be needed for using leftover body material for scientific purposes. BMJ 325:648-651

25. Angus AG et al (2010) Requirement of cellular DDX3 for hepatitis $C$ virus replication is unrelated to its interaction with the viral core protein. J Gen Virol 91:122-132

26. Yedavalli VS et al (2004) Requirement of DDX3 DEAD box RNA helicase for HIV-1 Rev-RRE export function. Cell 119:381-392

27. Budczies $\mathbf{J}$ et al (2012) Cutoff finder: a comprehensive and straightforward web application enabling rapid biomarker cutoff optimization. PloS One 7:e51862

28. Wu DW et al (2014) DDX3 loss by p53 inactivation promotes tumor malignancy via the MDM2/Slug/E-cadherin pathway and poor patient outcome in non-small-cell lung cancer. Oncogene 33:1515-1526

29. Chang PC et al (2006) DDX3, a DEAD box RNA helicase, is deregulated in hepatitis virus-associated hepatocellular carcinoma and is involved in cell growth control. Oncogene 25:1991-2003

30. Heerma van Voss MR et al (2015) DDX3 has divergent roles in head and neck squamous cell carcinomas in smoking versus nonsmoking patients. Oral Dis 21:270-271

31. Pugh TJ et al (2012) Medulloblastoma exome sequencing uncovers subtype-specific somatic mutations. Nature 488:106-110

32. Seiwert TY et al (2015) Integrative and comparative genomic analysis of HPV-positive and HPV-negative head and neck squamous cell carcinomas. Clin Cancer Res 21:632-641

33. Ojha $\mathrm{J}$ et al (2015) Identification of recurrent truncated DDX3X mutations in chronic lymphocytic leukaemia. $\mathrm{Br} \mathrm{J}$ Haematol 169:445-448

34. Wang L et al (2011) SF3B1 and other novel cancer genes in chronic lymphocytic leukemia. N Engl J Med 365:2497-2506

35. Nik-Zainal S et al (2016) Landscape of somatic mutations in 560 breast cancer whole-genome sequences. Nature 534:47-54

36. Topisirovic I et al (2009) Molecular dissection of the eukaryotic initiation factor 4E (eIF4E) export-competent RNP. EMBO J 28:1087-1098

37. Andersen JS et al (2002) Directed proteomic analysis of the human nucleolus. Curr Biol 12:1-11

38. Anders CK et al (2011) The prognostic contribution of clinical breast cancer subtype, age, and race among patients with breast cancer brain metastases. Cancer 117:1602-1611

39. Nguyen DX et al (2009) WNT/TCF signaling through LEF1 and HOXB9 mediates lung adenocarcinoma metastasis. Cell $138: 51-62$ 
40. Pukrop T et al (2010) Microglia promote colonization of brain tissue by breast cancer cells in a Wnt-dependent way. Glia 58:1477-1489

41. Cruciat CM et al (2013) RNA helicase DDX3 is a regulatory subunit of casein kinase 1 in Wnt-beta-catenin signaling. Science 339:1436-1441

42. McMullin RP et al (2014) A BRCA1 deficient-like signature is enriched in breast cancer brain metastases and predicts DNA damage-induced poly (ADP-ribose) polymerase inhibitor sensitivity. Breast Cancer Res 16:R25

43. Woditschka $\mathrm{S}$ et al (2014) DNA double-strand break repair genes and oxidative damage in brain metastasis of breast cancer. J Natl Cancer Inst 106:dju145

44. Berghoff AS et al (2015) Differential role of angiogenesis and tumour cell proliferation in brain metastases according to primary tumour type: analysis of 639 cases. Neuropathol Appl Neurobiol 41:e41-e55

45. Bol GM et al (2013) Expression of the RNA helicase DDX3 and the hypoxia response in breast cancer. PloS One 8:e63548
46. Dupuy F et al (2015) PDK1-dependent metabolic reprogramming dictates metastatic potential in breast cancer. Cell Metab 22:577-589

47. Chen EI et al (2007) Adaptation of energy metabolism in breast cancer brain metastases. Cancer Res 67:1472-1486

48. Palmieri D et al (2009) Analyses of resected human brain metastases of breast cancer reveal the association between upregulation of hexokinase 2 and poor prognosis. Mol Cancer Res $7: 1438-1445$

49. Heitz F et al (2009) Triple-negative and HER2-overexpressing breast cancers exhibit an elevated risk and an earlier occurrence of cerebral metastases. Eur J Cancer 45:2792-2798

50. Bol GM, Xie M, Raman V (2015) DDX3, a potential target for cancer treatment. Mol Cancer 14:188

51. Steeg PS, Camphausen KA, Smith QR (2011) Brain metastases as preventive and therapeutic targets. Nat Rev Cancer $11: 352-363$ 